\title{
Motricidad comunitaria: representaciones sociales en actores de la práctica pedagogíca Instituto de educación física*
}

\author{
Verónica Ochoa Patiño** \\ Martha Nancy Arias Henao***
}

Recibido: septiembre 15 de 2014 • Evaluado: noviembre 11 de 2014

Aceptado: noviembre 24 de 2014

\section{Resumen}

Preguntar por la Representación Social de la Motricidad Comunitaria es ir tras la comprensión, interpretación y actuación que comparten las personas que fueron indagadas. El estudio interrogó a dieciocho actores de la Práctica Pedagógica del Énfasis en Motricidad Comunitaria del nivel X del semestre 2013-I: tres profesores, diez estudiantes, tres cooperadores y dos integrantes de comités. Se encontró que en los participantes, se halla anclada una Representación Social de la Motricidad como dimensión humana. Existe un entendimiento de Comunidad desde el enfoque de la relación-interacción. Por último, para los participantes, la Motricidad Comunitaria se mueve entre Actividad Física para la Salud y énfasis de formación del que hacen parte prácticas que se hallan por fuera de las prácticas escolares, administrativas y del entrenamiento deportivo.

Palabras clave: Salud, actividad física, integración de la comunidad.

\footnotetext{
* Este artículo es producto de la investigación Representaciones Sociales de la Motricidad Comunitaria en procesos de Práctica Pedagógica, Nivel X Énfasis en Motricidad Comunitaria y su relación con el Modelo Curricular del Instituto Universitario de Educación Física de la Universidad de Antioquia. Desarrollado entre agosto de 2012 y julio de 2014. Financiado por el Comité para el Desarrollo de la Investigación - CODI - de la Universidad de Antioquia. Community Motor Function: The Social Representation in Actors of the Pedagogical Practice Institute of Physical Education.

** Magíster en Salud Colectiva. Docente - Investigadora de la Universidad de Antioquia, Colombia. Grupo de Investigación Estudios en Educación Corporal. Correo: veronica.ochoa@udea.edu.co

*** Magíster Psicología del Deporte y del Ejercicio Físico. Docente - Investigadora de la Universidad de Antioquia, Colombia. Correo: martha.arias@udea.edu.co
} 


\section{Community motor function: the social representation in actors of the pedagogical practice institute of physical education}

Abstract

Asking about the Social Representation of Community Motor Function is to seek the understanding, interpretation, and performance shared by the people who were surveyed. The study questioned eighteen actors of the Pedagogical Practice of the Emphasis on Community Motor Function - tenth level of the first term, 2013-I: three teachers, ten students, three cooperators, and two committee members. It was found that a Social Representation of Motor Function as a human dimension is rooted among participants. There is an understanding of Community from the relationship-interaction approach. Finally, for participants, Community Motor Function ranges over Physical Activity for Health and training emphasis. Some of its practices are outside the school, administrative, and sport training practices.

Keywords: Health, physical activity, community integration. 


\section{Introducción}

En el marco de la implementación de la Reforma Curricular del Instituto Universitario de Educación Física de la Universidad de Antioquia (Colombia) en el año 1999, se define la Motricidad como campo de formación y la Práctica Pedagógica como eje transversal en el Plan de Formación. Con sus tres etapas: básica, específica y énfasis, la Práctica Pedagógica está dirigida al desarrollo de habilidades para el desempeño profesional y a relacionar la formación con las necesidades e intereses de la sociedad. En los niveles IX y X, la Práctica Pedagógica se lleva a cabo en uno de cuatro énfasis: Motricidad Comunitaria, Entrenamiento Deportivo, Administración y Gestión y Escolar en la Educación Formal.

En torno al Énfasis en Motricidad Comunitaria han existido, en estos catorce años, diferentes intervenciones en contextos igualmente diversos: propuestas que ven el ejercicio como contributivo a la cura de la enfermedad, procesos de desarrollo local y formación comunitaria y ciudadana; iniciativas enfocadas a contrarrestar el sedentarismo y a promocionar la práctica masiva de la actividad física, proyectos centrados en la actividad física para el mejoramiento de la condición física y, en este sentido, mantener la salud y garantizar el bienestar; o buscando programas con perspectiva de género, condición de discapacidad, reclusión y desplazamiento.

En años recientes se ha abierto la discusión sobre qué se entiende por Motricidad Comunitaria y qué la constituye. Una de las razones para generar discusión es que, pese a la aceptación de ésta nominación como un escenario de práctica, intervención y desarrollo profesional, y que se tienen definidos unos contextos de acción, al parecer no están claramente precisados sus objetivos, los cuales hoy se confunden y entremezclan con los expuestos en otros escenarios de práctica. Otro asunto de interés es la discusión generada por la noción del término "comunitario", lo cual hace suponer a un importante grupo de profesores y estudiantes que el concepto de comunitario alude a un campo exclusivo de profesionales de las ciencias sociales. Por último, pese a que esta propuesta curricular lleva varios años, aún no hay un desarrollo epistemológico de la Motricidad Comunitaria y una postura académica colectiva que permita su delimitación.

Por ello consideramos que acercarnos a la Representación Social de la Motricidad Comunitaria a través de los actores (profesores, estudiantes, cooperadores e integrantes de los comités de práctica y currículo) que con 
sus voces trazan sus experiencias en la Práctica, hará hablar a la Motricidad Comunitaria, haciéndose visible para ella misma y para la comunidad académica del Instituto Universitario de Educación Física, enfrentándonos con el problema del indeterminismo conceptual, que intentará ser superado desde la mirada a las intersubjetividades que en el Currículo circulan con respecto a éste término. Por otro lado, permitirá precisar sus objetivos y conocer si efectivamente la acción comunitaria traspasa las fronteras de las disciplinas que tradicionalmente la han tratado. Por último, contribuirá al necesario desarrollo epistemológico de la Motricidad Comunitaria y precisará la postura académica colectiva que permitirá su delimitación.

Representación Social es: "un concepto híbrido donde confluyen nociones de origen sociológico, tales como la de cultura, o la de ideología, y nociones de procedencia psicológica, tales como la de imagen o la de pensamiento” (Ibáñez, 2001, p.170).

Las representaciones sociales conciernen al conocimiento del sentido común, que se pone a disposición en la experiencia cotidiana; son programas de percepción, construcciones con estatus de teoría ingenua, que sirven de guía para la acción e instrumento de lectura de la realidad; sistemas de significaciones que permiten interpretar el curso de los acontecimientos y las relaciones sociales; que expresan la relación que los individuos y los grupos mantienen con el mundo y los otros; que son forjadas en la interacción y el contacto con los discursos que circulan en el espacio público; que están inscritas en el lenguaje y las prácticas; y que funcionan como un lenguaje en razón de su función simbólica y de los marcos que proporcionan para codificar y categorizar lo que compone el universo de la vida (Jodelet, 2000, p.10).

La información, el campo representacional y la actitud son los elementos constituyentes de la Representación Social. "La información concierne a la organización de los conocimientos que tiene una persona o grupo sobre un objeto o situación determinada" (Araya, 2002, p. 40), lo que permite entenderlo, explicarlo y direccionar la respuesta hacia él.

La información proviene del contacto directo con el objeto y la práctica: "Esta información sobre el objeto varía en calidad, cantidad y precisión, según pertinencias grupales y ubicaciones sociales, influyendo así en el tiempo de representación que se forma" (Colina, 2000, p. 50). "El campo 
representacional expresa, la ordenación y jerarquización de los elementos que configuran el contenido de la representación” (Ibáñez, 2001, p. 185); “se construye alrededor del núcleo figurativo, parte más sólida y estable de la representación, ejerce una función organizadora que se construye a través del proceso de objetivación, proviene de la transformación de los diversos contenidos conceptuales relacionados con el objeto" (Colina, 2000, p. 50). La actitud, entre tanto, es: "la disposición más o menos favorable o desfavorable que tiene una persona hacia el objeto de la representación” (Ibáñez, 2001, p.184) en términos de motivación, comportamiento y gusto hacia él.

Motricidad: "es una categoría compleja y es sustentada desde las corrientes fenomenológica y de la complejidad, como también desde las ciencias sociales y humanas" (Benjumea, 2004, p. 10). Benjumea et al. (2004) la define como "un fenómeno donde confluyen características que trascienden los procesos biológicos e incorporan aspectos subjetivos de la complejidad humana como la cultura, lo social, lo simbólico y lo afectivo entre otros" (p. 10). Para Trigo y Rey (2000), "la dimensión conceptual de la motricidad excede el simple proceso "espacio-tem-poral”, para situarse en un proceso de complejidad humana: cultural, simbólico, social, volitivo, afectivo. Intelectual y por supuesto motor" (p. 94). La Motricidad se nutre de los aportes de Merleau Ponty con su "cuerpo sujeto" y se expresa, en la actualidad, en respuesta a los estudios adelantados por el profesor Manuel Sergio, como una nueva ciencia denominada Ciencia de la Motricidad Humana. Como ciencia plantea que es posible integrar la cualidad de movimiento que le es propia a todo ser vivo (en este caso al humano), con sus propias intenciones de comunicación e interrelación, que, siendo social por naturaleza, es decir comunitario, ha de valerse de esa interrelación, para sobrevivir, ser exitoso dentro de las sociedades a las cuales pertenece y dar cuenta de su existencia corporal y de su condición de humanidad.

Se evidencia que la motricidad asume como uno de sus componentes el movimiento (entendido como ejecución técnica) pero lo trasciende a fenómenos más integrales y complejos en la comprensión del ser, dado que es una expresión potencial del ser humano quien, por medio de las expresiones motrices, desarrolla la capacidad de relacionarse consigo mismo, con los otros y con el mundo físico; transmite y recrea valores determinados cultural, geográfica, política e históricamente (Benjumea, 2010, p. 150). 
La motricidad se expresa visiblemente a través del cuerpo en la funcionalidad sinérgica de órganos y sistemas que, con la necesaria participación de la propia voluntad, la intención y el propósito, hacen que el hombre transcienda y se proyecte a sí mismo, a los demás y a su comunidad en su total dimensión. Por eso, el papel de la motricidad humana no puede estar reducido a las manifestaciones técnicas de la motricidad, ni a aquellas que se relacionen con la producción, el rendimiento y la funcionalidad; por el contrario, debe trascender hacia expresión de la corporeidad como forma de vida, como manifestación de sentido que, pese a estar construido desde las relaciones con el otro y lo otro, se expresan de forma individual y los hacen ser lo que somos y podremos llegar a ser.

La motricidad ocurre en las interrelaciones que el hombre lleva a cabo con y en su ambiente, que necesariamente lo hace ponerse en comunicación con los objetos y las personas que lo conforman; de esta manera responde a las situaciones que se recrean allí y procura otras. Por ello se puede afirmar que la motricidad se expresa en tres ámbitos de interrelación hombre - ambiente: el ámbito de lo cotidiano, el ámbito de lo ocupacional y el ámbito del ocio, el tiempo libre y la recreación, las cuales, teniendo un objetivo concreto o no, son circunstanciales o predispuestas por el hombre.

Las definiciones de comunidad pueden ser reunidas en cuatro conjuntos: 1) La idea de localidad o área geográfica; se trata de todas las definiciones que tienen primordialmente en cuenta los límites geográficos o la influencia de los factores físicos sobre las relaciones sociales. 2) Estructura social de un grupo, es decir, el conjunto de relaciones sociales estudiándose las instituciones del mismo y los problemas de los roles, status y clases sociales que se dan en su interior. 3) Desde los sentimientos o conciencia de pertenencia que las caracteriza. 4) Equiparación a sociedad (Ander-Egg, 1990 citado por Aguilar, 2001, p. 25).

De manera más reciente, Maya pone de manifiesto “el surgimiento de nuevas formas comunitarias, caracterizadas por el auge del individualismo y la personalización de las prácticas sociales" (Maya, 2002; Smith \& Kollock, 1999; Wellman, 2001, citados por Maya, 2004, p.188) que hacen ver la comunidad no desde el sentido de proximidad en un espacio, sino "como grupo relacional, definiendo la comunidad con base en las relaciones interpersonales 
(y en el sentido de pertenencia resultante); por ejemplo, el grupo de autoayuda" (Maya, 2004, p. 190), donde los participantes desarrollaban cierto sentido de comunidad. En sentido:

...el tiempo contemporáneo define un nuevo tipo de comunidad. Su origen está en las actividades surgidas en los momentos de ocio, en actividades de consumo y, en general, en todas aquellas prácticas nacidas del mundo privado vivido como refugio, presa del imperio del individualismo" (Gurrutxaga, 1993, p. 219), "esto conduce a percibir la comunidad desde el lado del sujeto y a considerarla como un producto social que surge en cualquier ambiente y contexto; puede tener carácter formalizado y duradero; ser estable pero poco formalizado o responder a necesidades del momento" (Gurrutxaga, 1993, p. 202).

\section{Metodología}

En esta investigación se hace escucha atenta a las voces de dieciocho actores de la Práctica Pedagógica del Énfasis en Motricidad Comunitaria del nivel X del semestre 2013-I del Instituto Universitario de Educación Física de la Universidad de Antioquia: tres profesores, diez estudiantes, tres cooperadores y dos integrantes de los comités. La selección fue intencionada, porque los criterios se ajustaban a la condición de estudiantes, profesores, cooperadores o miembros de lo que para ese semestre eran los Centros de Práctica en Motricidad Comunitaria y los comités. De un universo de aproximadamente 40 personas respondieron al llamado 18 participantes.

A los participantes se les indagó por la motricidad, la comunidad y, en este sentido, por la Motricidad Comunitaria, como una manera de acercamiento a los contextos y a la realidad social que se configura en la interacción de los actores cuando se ponen en escena en la Práctica y se afectan por ella misma, asunto que, de manera no intencional, va configurando una manera socialmente identificable de entender y hacer vida lo que para ellos es la Motricidad Comunitaria, es decir, va configurando una Representación Social de ésta.

La manera de obrar obedece a una investigación hermenéutico - interpretativa. A la manera de Gadamer, este tipo de investigación ve en la realidad social un texto susceptible de disquisición. 
El problema de la hermenéutica va más allá de las fronteras impuestas por el concepto de método de la ciencia moderna. Comprender e interpretar textos no es sólo una instancia científica, sino que pertenece con toda evidencia a la experiencia humana del mundo. (Gadamer, 1993, p. 23).

Por otro lado, el estudio toma como perspectiva teórica el Interaccionismo Simbólico.

La concepción interaccionista se fundamenta en el principio de que el comportamiento humano es autodirigido y observable en dos sentidos: el simbólico y el interaccional. Esto permite a cualquier ser humano planificar y dirigir sus acciones en relación con los otros y otorgar significado a los objetos que utiliza para realizar sus planes. Esta concepción asume la vida social como un consentido establecido en la interrelación, con lo cual el sentido atribuido a las acciones es manipulado, redefinido y modificado a través de un proceso interpretativo en sentido del grupo (De Souza, 1997, p. 47).

El Interaccionismo Simbólico expresa que la capacidad de pensamiento propio de los seres humanos está modelada por la interacción social, en la cual las personas aprenden los significados y los símbolos que les permiten ejercer esta capacidad y actuar e interactuar de una manera distintivamente humana. Las personas tienen la capacidad de modificar y alterar los significados y símbolos en la acción y en la interacción sobre la base de la interpretación de las situaciones. La modificaciones son introducidas gracias a la capacidad que tienen las personas de interactuar consigo mismas, lo que les permite examinar los posibles cursos de acción, valorar y elegir. Las pautas de acción e interacción dice el interaccionismo simbólico, constituyen los grupos y las sociedades (Blumer, 1996a; Manis \& Meltzer, 1978; Rose, 1962, citados por Ritzer, 1993, p. 271).

En correspondencia, el estudio apeló a la técnica de la entrevista semiestructurada. La recolección de información se realizó hasta obtener la saturación de categorías. De ésta manera se procuró adentrarse en el mundo que cada uno de los actores construye alrededor de la Práctica de énfasis en Motricidad Comunitaria y de esta forma se interpretó las "Representaciones Sociales" 
que circulan sobre la Motricidad Comunitaria, como objeto de interés de esta investigación. Las entrevistas fueron grabadas, previa firma del consentimiento informado por parte de los participantes y, luego transcritas. Se asignó a cada participante un código en letra y número y cada respuesta fue identificada con la letra $\mathbf{p}$ de pregunta y el número correspondiente a la pregunta, ejemplo E1ps (Estudiante1-pregunta 2).

Para el análisis, se alió a la metodología de la Teoría Fundamentada. "Ésta metodología se apoya teóricamente del Interaccionismo Simbólico" (Murcia \& Jaramillo, 2000, p.57), y se traza como objetivo "generar teoría derivada de datos recopilados de manera sistemática y analizados por medio de un proceso de investigación" (Strauss \&, Corbin 2002, p.13, De la Cuesta, 2006, p.137). La Teoría Fundamentada ofrece un procedimiento analítico básico denominado codificación en tres momentos: descriptiva, axial y selectiva y en su proceso recurre esencialmente a tres técnicas: la conceptualización, los memos y los diagramas.

\section{Resultados}

Al preguntar por la Representación Social, estamos tras el significado ${ }^{1}$ : una comprensión, interpretación y actuación compartida por los participantes de la investigación frente a la Motricidad Comunitaria.

Cuando un grupo social tiene una Representación Social sobre algo, se dice que dicha representación está anclada. Para que ello ocurra, la Representación Social pasa por un momento de concreción denominado objetivación, proceso mediante el cual la Motricidad Comunitaria se transforma en algo concreto, material y con sentido para el sujeto. La selección y descontextualización de los elementos que conforman la Motricidad Comunitaria, la formación de la parte central de la Representación o del modelo o núcleo figurativo y la naturalización (en sujeto) de dicho núcleo, son los subprocesos que la constituyen.

1 Cuando los sujetos actúan e interactúan forman comprensiones comunes a través de la comunicación y, en última instancia, un punto de vista común sobre sus experiencias (Ritzer, 1993, p.432). 
Con el anclaje, esos sentidos — vía procesos de interacción e intersubjetividades - pasan al plano de la significancia para la colectividad, uniéndose a su marco de referencia y posibilitando decodificar la realidad que, con respecto a la Motricidad Comunitaria, se va construyendo y actuar sobre ella. Gracias al anclaje, para el grupo social la Motricidad Comunitaria se carga de valor, adquiere un significado, cobra importancia, se establece como marco de referencia, se inscribe en el sistema de valores, se perfila como un instrumento, empieza a hacer parte de las preferencias y las interrelaciones; además, las informaciones contenidas sobre la Motricidad Comunitaria se interpretan de manera fácil integrándose al sistema de pensamientos, valores y funciones que el grupo social ha dado a ésta a través de su representación.

Las experiencias de la vida cotidiana y académica, los procesos conversacionales ocurridos en las interacciones sociales, los simbolismos que se hallan en el mundo social y la información académica que circula, son nutrientes para la Representación Social que, sobre Motricidad, Comunidad y Motricidad Comunitaria, tienen los participantes. Al ser indagados, algunos participantes destacaron cómo sus experiencias personales, familiares y deportivas estuvieron siempre cercanas a procesos barriales y contextos grupales. Para la mayoría de ellos, las actividades físico-deportivas han sido, y siguen siendo, parte constitutiva de su vida. Otros destacan cómo los procesos formativos y profesionales en la Licenciatura en Educación Física han estado vinculados a programas institucionales o estatales dirigidos a poblaciones específicas, con fines de movilización y transformación personal o social, gratuidad y voluntariado. Se destaca cómo algunos de los participantes mencionan sin detalles adicionales, por un lado, los ejes que vinculan la motricidad, la promoción de la salud y la comunidad y, por otro lado, a los grupos de investigación, especialmente Cultura Somática y Ciencias Aplicadas a la Actividad Física y el Deporte².

Los juicios, razonamientos y conceptos expresados por los participantes que reflejan la Representación Social existente sobre la Motricidad Comunitaria y sus elementos, se hallan en un proceso continuo de abstracción y generalización, lo que permite, por un lado, afianzar, decodificar y transformar los modos de pensar y actuar con respecto a la Motricidad, la Comunidad y la Motricidad Comunitaria, y, por otro lado, fijar la

2 Dos, de los cinco grupos de investigación del Instituto Universitario de Educación Física. 
información, el campo representacional y la actitud que, como elementos de la Representación Social, muestran en definitiva la Representación Social que sobre la Motricidad Comunitaria tienen los participantes del estudio.

\section{Representación social de la motricidad}

El estudio evidencia que en los participantes se halla anclada una Representación Social de la Motricidad, como dimensión humana.

La reflexión ontológica que considera la motricidad como dimensión bumana entiende que la primera función del organismo humano es la organizadora, desde su función orgánica y de convivencia social. Pero el organismo tiene una segunda función, la que le confiere 'presencia real', es la Corporeidad, el organismo organizado y solidario en que precisamente toma cuerpo la realidad viva del viviente, y que se comporta como dimensión (Benjumea, 2010, p.165). Como dimensión humana, la Motricidad es una realidad tangible en la que se conjugan múltiples fenómenos de orden orgánico y sociocultural que interactúan en su génesis y en su manifestación, consolidándose como acto de comunicación y expresión humana, acto que nos diferencia de las demás especies por el carácter de intencionalidad que emerge en la acción que, además, se forma a partir de las interacciones e interrelaciones con el otro, los otros, el entorno y todas las situaciones que se generan o confluyen en el ser humano (Benjumea, 2010, p.192).

Si bien es cierto el término movimiento, desde algunas perspectivas filosóficas (especialmente de corte dialéctico), tiene la misma significación que cambio, devenir y transformación, su uso obligado refiere la traslación y el desplazamiento. Este modo, que en campos como la Educación Física es convencional, está inspirado en las modernas ciencias naturales, las cuales, por un lado, negaron el problema del cambio ontológico y redujeron el concepto al cambio o al desplazamiento de partículas en el espacio, en correspondencia a leyes y principios, y, por otro lado, presentaron al cuerpo como una estructura física y material susceptible de desplazamiento y uso.

Lo que han concebido los participantes en forma cartesiana y mecanicista con respecto al cuerpo, en razón de uso y práctica, y que se expresa 
en una idea de movimiento anclada a las ciencias naturales, toma un giro al que han llamado Motricidad y que si bien es cierto lo definen como movimiento, no lo evocan sólo y exclusivamente al desplazamiento de una cosa (o fracción de ésta) llamada cuerpo, sino que lo evocan ontológicamente ${ }^{3}$, ello es, lo cargan de cualidades y sentidos. De acuerdo con Merleau-Ponty (1975, p.156), "la motricidad no es como una criada de la consciencia, que transporta el cuerpo a aquel punto del espacio que primero nos habríamos representado". "Deja de ser la simple consciencia de mis cambios de lugar presentes o próximos para convertirse en la función que, en cada momento, establece unos patrones de magnitud, en la amplitud variable de mi ser-delmundo" (Merleau Ponty, 1975, p.226).

Según los participantes, este movimiento tiene, en sí mismo, una carga comunicativa, relacional y expresiva ${ }^{4}$. Esa relación le permite a la persona hacerse a sí misma, devenirse, cambiarse, transformarse. Los datos nos hacen ver que implícitamente se halla un reconocimiento al cambio ontológico, ello es, al movimiento, a la transformación, al devenir que se sucede en el ser humano al expresarse en éste y en la relación con el otro y lo otro, la dimensión humana de la Motricidad vía intersubjetividades ${ }^{5}$.

3 A la manera como es comprendida la Ontología por pensadores del siglo XX, como Heidegger, ella se refiere al ser en cuanto ser, pero no como una mera entidad formal, ni como una existencia, sino como aquello que hace posibles las existencias. Ontología "como doctrina del ser" (Heidegger, 2008, p.17), como categoría de existir y referida al ser humano.

4 Para Husserl, "nada del sentido recibido de específica corporalidad viva puede realizarse originalmente en mi esfera primordial, en mi cuerpo vivo y físico" (Husserl, 1996, p.177).

En ella (en mi esfera primordial) destaca mi cuerpo orgánico por su unicidad, como único cuerpo físico que además se constituye como soma. Si en mi primordialidad se destaca otro cuerpo físico, entonces la similitud entre mi cuerpo físico y el ajeno fundará la motivación de la transferencia de sentido de mi cuerpo orgánico a ese otro cuerpo físico como un segundo cuerpo orgánico. Tenemos, así, otro yo trascendental que gobierna su cuerpo viviente, el que remite a mi cuerpo orgánico como instauración originaria. Y ésta es otra particularidad de la constitución del otro, pues, en oposición a las apercepciones cósicas, en la apercepción del otro el original soy yo mismo; dicho de otro modo, el original es constantemente "presente y viviente (Chu, 2002, p.175).

5 Para Merleau Ponty: Si, reflexionando sobre la esencia de la subjetividad, la encuentro vinculada a la del cuerpo y a la del mundo, es que mi existencia como subjetividad no forma más que una sola cosa con mi existencia como cuerpo y con la existencia del mundo y que, finalmente, el sujeto que yo soy, tomado concretamente, es inseparable de este cuerpo y de este mundo. El mundo y el cuerpo ontológicos que encontramos en la mismísima médula del sujeto no son el mundo en idea o el cuerpo en idea, es el mismo mundo contraído en punto de presa global, es el mismo cuerpo como cuerpo-cognoscente" (Merleau Ponty, 1975:417) 
Hablar de motricidad es hablar de movimiento intencionado, consciente, voluntario, planeado, acto pensado que se hace con un sentido $\mathrm{y}$ tiene una finalidad. $\mathrm{P} 1 \mathrm{p} 2$

Son los movimientos que puede realizar el ser humano, en relación a sus habilidades y capacidades, en pro siempre de un objetivo en específico. E2p2

Es un tipo de actividad que tiene una intención, que puede ser de muchas índoles: cotidiano, recreativo, de salud, que siempre está en nosotros, que tiene que ver con lo que se siente, lo que se trasmite a los otros, que tiene que ver con elementos físicos, elementos psicológicos y elementos de los entornos en los que se desenvuelve el ser humano. E3p2

Uno como persona, tiene varios campos o varias dimensiones sobre las cuales está y una de ellas es la motricidad, es una dimensión del ser, del ser humano. Cuando hablamos de motricidad lo vemos como una posibilidad, incluso de relacionamiento con el otro, no la vemos sólo como el hecho de venir a hacer ejercicio y sudar. La vemos como una posibilidad de que eso nos ayude a mejorar la calidad de vida de aportar al bienestar de las personas. CO3p2

Movimiento es lo que primero se le viene a uno a la cabeza, pero creo que cuando uno habla de motricidad son más elementos, o sea es el movimiento pero son las relaciones, el mismo cuerpo es lo que hace que uno se mueva, es más bien que el movimiento hace parte de la motricidad. $\mathrm{CO} 2 \mathrm{p} 2$

Motricidad es un movimiento intencional y la intencionalidad es formar. La motricidad me la da mi humanidad para diferenciarla del movimiento motriz. Cuando digo motricidad es más desde la humanidad. CP1p2

Si bien es cierto los participantes, al referir Motricidad no hacen alusión a la Actividad Física (y a la Educación Física), sí lo hacen al ser indagados por la Motricidad Comunitaria. Ellos refieren a Actividad Física como generadora de procesos de trasformación individual, colectiva e incluso de sociopolítica, lo que hace ver, de manera implícita, a la Motricidad como parte de la construcción intersubjetiva del ser a lo largo de su existencia. 


\section{Representación social de comunidad}

El estudio muestra que en los participantes se halla anclada una Representación Social de Comunidad con los siguientes elementos: conjunto, grupo o agregación de personas cuyos integrantes, compartiendo cualidades, intereses, servicios o equipamiento comunes, interactúan socialmente estableciendo lazos y relacionamientos múltiples e intensos.

Para mí comunidad es una población específica, un grupo poblacional... Es población particular, es grupo grande, son los barrios, los grupos de investigación, los grupos deportivos, políticos o culturales... Grupo de personas sin importar qué diferentes sean... Es la aglomeración de personas... P1p3

Lo relaciono siempre con personas, con pequeñas sociedades, diversas poblaciones: adultos mayores, jóvenes, gestantes, personas en condición de discapacidad, de desplazamiento, infinidad de subgrupos... Un lugar, un pueblo, una ciudad, un barrio, una cuadra pueden ser una comunidad. E2p3

Una comunidad se caracteriza por algunas especificaciones: ser negra, indígena, blanca, mulata, deportiva, académica, todas con un vínculo filial... Hay algo que las relaciona: la actividad que desarrollan, el lugar que en el cual están, las características físicas... Que comparten en un espacio, un objetivo, una meta, un fin, una forma de organización, algo en común P2p3

La comunidad es donde la gente comparte sus experiencias, donde permanecen bajo unas reglas, un orden, para la relación con el otro y que permite una sana convivencia. E6p3

La comunidad son aquellas personas que se interesan por venir a aprender un poquito de lo que aquí les podemos ofrecer, desde aquí no es tan vista como esa cosa organizada más desde el ámbito político sino como el ciudadano a pie que se junta con otros por el interés de acceder a algún programa que estemos ofreciendo. $\mathrm{CO} 2 \mathrm{p} 3$

Concepto de las ciencias sociales más desde lo antropológico, desde lo sociológico que identifica grupos humanos acogidos por historias comunes por unos rasgos identitarios claramente definidos y con unos intereses colectivos. CC2p3 
En este sentido, la Representación Social de Comunidad es consecuente con un entendimiento de Comunidad desde el enfoque de la relación-interacción, dejando de lado el convencional enfoque de escenario-territorio y el más reciente enfoque del sentido-significado. Vislumbraremos igualmente que para los participantes la Motricidad Comunitaria es Actividad Física y Salud, y en este sentido la Comunidad, para los participantes, es objeto de intervención a través de programas sociales y de salud. En consecuencia, Comunidad sigue siendo el concepto empleado para designar, tres cosas: el objeto de atención, el nivel de intervención o la estrategia de acción. (Canals, 1991, citado por Aguilar, 2001, p.27).

\section{Representación social de motricidad comunitaria}

El estudio muestra que en los participantes se hallan anclada una representación social de la Motricidad Comunitaria como Actividad Física y Salud, y en este sentido, la vinculan con la realización de prácticas de ejercitación, deportivas y/o lúdicas en y con grupos institucionalizados. También se analiza como fluctúa esta representación social entre los dos paradigmas existentes sobre Actividad Física. Uno, el paradigma que considera la Actividad Física como:

...interacción con el entorno, utilizando el movimiento corporal, desde una óptica funcional; el movimiento corporal de cualquier tipo, producido por la contracción muscular que conduce a un incremento del gasto energético; una práctica social ya que es un medio para interrelacionarse con otras personas, y a su vez una experiencia personal que permite a las personas conocerse a ellas mismas y aceptar las limitaciones (González, 2004, p.72; Devís, 2001, p.299).

Para los participantes pensar la Motricidad Comunitaria así, es ver la Motricidad más allá del soma, es decir más allá de una expresión biológico-funcional, es ver la Motricidad como como cualidad o dimensión humana ${ }^{6}$ y la actividad física como una práctica para el bienestar.

6 Ver Representación social de la Motricidad en este documento. 
Dos, el paradigma liderado por la Organización Mundial de la Salud (OMS) que define la Actividad Física como cualquier movimiento corporal que se da por la musculatura esquelética, generando gasto energético, que es determinante en el equilibrio calórico y en el control del peso debido al gasto de energía que produce. Esta perspectiva de índole sanitaria que lidera por la OMS, ha orientado las políticas, las estrategias y los programas que tradicionalmente han implementado los gobiernos e instituciones bajo lemas como: Educación para la Salud, Vida Activa y Buenas Conductas Alimentarias, cuyos objetivos fundamentales son la disminución de la morbimortalidad en la población, ello en consideración a que "promover actividad física es esencial para prevenir las enfermedades y promover la salud, una alta calidad de vida y el bienestar general" (OMS, 2008, p.1).

Como pude observarse, ambas perspectivas de la Actividad Física refieren a la calidad de vida y al bienestar. "La expresión calidad de vida es utilizada tanto en la literatura científica como en el lenguaje corriente" (Rodado \& Grijalba, 2001, p.25), para referir en conjunto, al sentimiento de complacencia o no que experimenta un sujeto ante las circunstancias de la vida. Este sentimiento está dado, en mayor o menor medida, por la satisfacción de necesidades de todo orden, materiales, sociales y espirituales. Así “aunque en calidad de vida, se evalúan los componentes del bienestar objetivo, se considera primordialmente la percepción y estimación de ese bienestar objetivo, el análisis de los procesos que conducen a esa satisfacción y los elementos integrantes de ésta" (Oblitas, 2006, p.249). En este sentido, la calidad de vida está relacionada con el bienestar, considerado como un "estado mental agradable o deseado de goce, y como la satisfacción de deseos o preferencias de las personas" (Rodado \& Grijalba, 2001, p. 28), también tiene que ver con "el cuidado del cuerpo, el planificar los ejercicios a realizar, el descansar lo necesario y el alimentarse adecuadamente" (González, 2004, p. 84, Devís, 2001, p. 300).

También los datos muestran como algunos participantes, refieren la Motricidad Comunitaria como un énfasis de formación del Licenciado en Educación Física en la Universidad de Antioquia; los participantes consideran que tal denominación ayuda a resolver, en términos prácticos, todo lo que se halla por fuera de los otros énfasis: Escolar, Entrenamiento Deportivo y Administración Deportiva, aunque, para algunos de los participantes, la Motricidad Comunitaria incluye elementos inherentes a ellos. 
La Motricidad Comunitaria es el trabajo que se hace con una población específica y con objetivos específicos. Tienen que ver con aspectos de lo motriz situados en un periodo del ciclo vital que va desde la gestación hasta la tercera edad. E3p1

Son las actividades encaminadas a un público general. Acciones o ejercicios que se hacen con los distintos grupos... Acciones de forma consciente en determinados grupos de la población... Acciones grupales que tienden a la promoción de la salud. E6p1

Son asuntos que tienen que ver con el movimiento y la comunidad, con los barrios, el municipio, la escuela, inscritos en el ámbito de lo que es la promoción de la salud y la prevención de la enfermedad. Aquellas expresiones motrices encaminadas hacia los valores, las habilidades personales, los hábitos saludables y las actividades de autoconocimiento. P2p1

Son todas aquellas prácticas que se realizan en grupos o con comunidades en los diferentes espacios, sea tanto desde el ámbito de la salud, como en el ámbito deportivo, como en el ámbito escolar, en los cuales tenga injerencia o pueda participar un educador físico. Motricidad Comunitaria es un programa que utiliza el movimiento intencionado en una población. Es la práctica de actividad física por gusto personal y socialización, cuando se da algo por gusto y se genera un espacio, una práctica, una conciencia, se lucha por una conciencia, un ideal, una idea, por llegar a un objetivo específico. P1p1

La Motricidad Comunitaria es la intervención que los educadores físicos hacen en un medio social, pero impactando en los problemas sociales de una comunidad específica. Lo que más ha afirmado a la motricidad comunitaria es la intervención en Actividad Física y Salud, pero que ahora con el nombre que ha renombrado a la motricidad comunitaria ha ampliado pues todo ese campo. CP1p1

Un escenario donde se visualiza una comunidad académica intentando construir nuevas formas de ver el campo, de comprenderlo, incluso de proponer nuevas prácticas, nuevas reflexiones. Un escenario social que demanda, que espera de este campo y, de los profesionales de este campo algo diferente a lo que se desarrolla en otros escenarios. CC1p1 


\section{Consideraciones finales: la motricidad comunitaria y el modelo curricular del Instituto de Educación Física}

En el Instituto Universitario de Educación Física, la acepción a Motricidad Comunitaria emergió aproximadamente en el año 1996. Para entonces estaban en auge, por un lado, todos los discursos de los profesores Manuel Sergio y Eugenia Trigo respecto a la Motricidad, y, por otro lado, el concepto de comunidad y el trabajo comunitario, y el Instituto no fue ajeno a ello. La participación de profesores y estudiantes en el denominado Movimiento Niño (proyecto que hacía parte de la Estrategia Municipios Saludables) en los municipios de Guatapé y Alejandría (Antioquia, Colombia) con propuestas desde el deporte, la lúdica y la actividad física para el fortalecimiento de la organización y la participación comunitaria y la recuperación del tejido social, abre la posibilidad para que dicha acepción empezara a naturalizarse entre la comunidad académica del Instituto.

Como un nuevo escenario, la Motricidad Comunitaria pasó a ser parte de los énfasis de formación, que reunió dos tipos prácticas: las circunscritas a lo que hasta en ese momento era la Actividad Física ${ }^{7}$ y las prácticas cuyo enfoque era netamente comunitario, es decir, prácticas inscritas en intervenciones a grupos poblacionales en condición de vulnerabilidad socio-política, cuyo objeto era el empoderamiento comunitario y la resiliencia de quienes hacían parte de ella.

Para el momento de este estudio, las cosas han seguido su curso natural de transformación, movimiento y cambio, y la Motricidad Comunitaria, respondiendo a los discursos de la Promoción de la Salud y la Prevención de la Enfermedad, se ha convertido en Actividad Física y Salud y ha perdido lo que le era natural: los procesos de organización y participación comunitaria con fines socio-políticos. Sin embargo, conserva lo que le es sustancial a ella y se halla expuesto con especial énfasis en la propuesta curricular del Instituto universitario de Educación Física de la Universidad de Antioquia: la trasformación social ${ }^{8}$.

7 Noción que desde políticas sanitarias, epidemiológicas y de salud pública toma más adelante un calificativo adicional y de salud.

8 A través de su currículo, el Instituto Universitario de Educación Física, asume la responsabilidad de aportar a la transformación social, cumpliendo con la doble función. 
Como proyecto cultural, el currículo del Instituto Universitario de Educación Física, su desarrollo, implementación y puesta en escena, ha contribuido de manera especial a la construcción de la Representación Social que sobre la Motricidad, la Comunidad y la Motricidad Comunitaria fue hallada en el estudio. Respecto a la Motricidad, se considera que existe una relación dialéctica entre la Representación Social de la Motricidad como dimensión humana y lo que expone el modelo pedagógico del Instituto universitario de Educación Física, el cual versa:

El modelo constructivista, concibe la Motricidad como expresión del potencial de lo humano la cual por medio de las expresiones motrices, desarrolla la capacidad de relacionarse consigo mismo, con los otros y con el mundo, la cual transmite y recrea valores determinados, cultural, geográfica, política e históricamente. La Motricidad como objeto de estudio de la Educación Física se centra en la promoción del ser humano y en la transformación social como objetivos fundamentales del proceso educativo. Puede decirse que la corporeidad es el sustrato que se construye a través de la motricidad. Se caracteriza por su naturaleza intencional, puesto que el ser humano, cuando se mueve, compromete todo su ser en cualquier expresión que realice y cada expresión posee un carácter simbólico que surge de su subjetividad y de la relación con otros y con el entorno (IUEF, 2002, p.13).

La Representación Social de comunidad se halla de manera implícita en lo que la propuesta curricular concibe con respecto a la Práctica Pedagógica:

La práctica pedagógica es fundamentalmente una búsqueda de la condición humana, un escenario donde se relaciona el propósito de formación con las necesidades e intereses de un contexto social determinado, donde se identifican problemas de orden científico, profesional y social, donde se crean propuestas pedagógicas innovadoras con el fin de aportar a la construcción de la episteme de la motricidad, y desde ésta aportar a la calidad de vida, la transformación

En primer lugar, personas que lideren el desarrollo cultural, difundan el saber socialmente construido y se construyan en comunidad académica. En segundo lugar, produciendo conocimiento, teniendo en cuenta la pluralidad, la interdisciplinariedad y la relación teoría y práctica, de tal manera, que permita gradualmente una mayor comprensión de la realidad y, a la vez, la generación de alternativas para la solución de los problemas de tipo científico y social de una comunidad determinada (IUEF, 2002, pp. 10-11). 
social y a su proyecto de vida, donde el trabajo de campo se centra, en una parte, en la participación en procesos de interacción y gestión pedagógica, relacionadas con las expresiones motrices artísticas, deportivas y comunitarias, de acuerdo al proceso vital humano (niños, adolescentes, adultos y viejos) y, en otra parte, en el diseño y la ejecución de un proyecto pedagógico que pretenden generar alternativas de solución de problemas de orden científico, profesional y social (IUEF, 2002, p.31).

Por último, desde la Guía del Diseño Curricular (IUEF, 2002, p. 21), la Motricidad Comunitaria se presenta como un énfasis de formación ${ }^{9}$ a desarrollarse en "un área específica de la motricidad", en "un escenario concreto llamado comunitario", y ello coincide con la segunda Representación Social de Motricidad Comunitaria que la hace ver como énfasis de formación ${ }^{10}$. Sin embargo, las interacciones comunicativas y las interacciones sucedidas entre los participantes, hacen ver que esta forma de entender la Motricidad Comunitaria permanece, pero alimentada con la idea de que en ella hacen presencia todas aquellas prácticas que se hallan por fuera de las prácticas escolares, las de entrenamiento deportivo o de administración deportiva y que posiblemente ese pensar hace parte de la fase de objetivación que lleva a la construcción de una Representación Social de la Motricidad Comunitaria tal vez diferente a la que exponen un grupo importante de participantes en esta investigación que la hacen ver como Actividad Física y Salud, lo que faltaría comprobar.

Pese a que en términos prácticos en el instituto Universitario de Educación Física la Motricidad Comunitaria hoy, se comprende como Actividad Física y Salud, ello es, la realización de prácticas de ejercitación, deportivas y/o lúdicas con grupos institucionalizados, el que los actores hallan referido a la Motricidad más allá del soma, como dimensión humana que evoca cambio ontológico, dota de un diferente sentido al significante Motricidad Comunitaria y ello en términos de la transformación social que busca la Universidad y el Instituto

9 La etapa de énfasis —último año de formación en la licenciatura- permite que el estudiante se ponga en contacto con un área específica de la motricidad. Su propósito es desarrollar proyectos de innovación pedagógica, con el fin de dar respuestas a necesidades de tipo social, disciplinar o profesional desde los escenarios escolar, deportivo, comunitario y administrativo.

10 La primera, hace ver a la Motricidad Comunitaria como Actividad Física y Salud. 
de Educación Física abre en un sentido político y académico perspectivas de actuación potencialmente contributivas a este fin.

\section{Referencias}

Aguilar, M. J. (2001). La participación comunitaria en salud. Rito o Realidad: evaluación de experiencias en atención primaria. España: Díaz de Santos.

Araya, S. (2002). Las representaciones sociales: Ejes para su discusión. Costa Rica: Facultad Latinoamericana de Ciencias Sociales.

Benjumea, M. (2004). La motricidad, corporeidad y pedagogía del movimiento en educación física. Un asunto que invita a la transdisciplinariedad. Brasil: Universidad Metodista de Piracicaba.

Benjumea, M. (2010). La Motricidad como dimensión humana. Un abordaje transdisciplinar. España: Léem.

Colina, C. (2000). De la teoría de las representaciones sociales a las mediaciones. Comunicación. Caracas, 110, 46-55.

Chu, M. (2002). El otro y la esfera primordial. Reflexiones sobre la intersubjetividad en las Meditaciones cartesianas. Perú: Pontificia Universidad Católica.

De La Cuesta, C. (2008). La teoría fundamentada como herramienta de análisis. Cultura de los cuidados. Alicante, 20, 136-140.

De souza, M.C. (1997). El desafío del conocimiento. Investigación cualitativa en salud. Buenos Aires: Lugar Editorial.

Devís, J. La educación física, el deporte y la salud en el siglo XXI. España: Editorial Marfil, 2001.

Gadamer, H.G. (1993). Verdad y Método. Fundamentos de una Hermenéutica. Salamanca: Ediciones Sígueme.

González, J. (2004). La actividad física orientada a la promoción de la salud. Escuela Abierta, 7, 73-96.

Gurrutxaga, A. (1993). El sentido moderno de la comunidad. Revista Española de Investigaciones Sociológicas, 64, 201-222.

Heidegger, M. (2008). Ontología hermenéutica de la facticidad. Madrid: Alianza Editorial. Husserl, E. (1996). Meditaciones Cartesianas. México: Fondo de Cultura Económica. Ibáñez, T. (2001). Psicología social construccionista. Guadalajara: Universidad de Guadalajara. 
Instituto Universitario de Educación Física -IUEF. (2002). Guía del Diseño Curricular. Medellín: El Instituto de Educación Física Universidad de Antioquia.

Jodelet, D. (2000). Develando la Cultura. México: Universidad Nacional Autónoma.

Maya, I. (2004). Sentido de comunidad y potenciación comunitaria. Apuntes de Psicología. España, 22(2), 187-211.

Merleau-Ponty, M. (1975). Fenomenología de la percepción. Barcelona: Ediciones Península.

Murcia, N. \& Jaramillo, L.G. (2000). Investigación cualitativa, la complementariedad etnográfica: propuesta desde la práctica reflexiva una guía posible para abordar estudios sociales. Manizales: Kinesis.

Oblitas, L.A. (2006). Psicología de la salud y calidad de vida. México: Thomson.

OMS. (2008). Aplicación de la estrategia mundial sobre régimen alimentario, actividad física y salud. Una guía de enfoques basados en población para incrementar niveles de actividad física. Organización Mundial de la Salud.

Ritzer, G. (1993). Teoría sociológica contemporánea. México: McGraw-Hill.

Rodado, C. \& Grijalba, E. (2001). La tierra cambia de piel: una visión integral de la calidad de vida. Colombia: Planeta.

Strauss, A. \& Corbin, J. (2002). Bases de la investigación cualitativa. Técnicas y procedimientos para desarrollar la teoría fundamentada. Medellín: Universidad de Antioquia.

Trigo, E. \& Rey, A. (2000). Motricidad... ¿Quién eres? Apunts. Catalunya, 59, 91-98. 